\title{
Phosphorylated low-density lipoprotein receptor-related protein 6 is prevalent in hippocampal progenitor cells and circuits of aged human hippocampus
}

\author{
Christopher P. Sullivan ${ }^{1,2^{\star}}$, Rosemary Elliott-Bryant ${ }^{1}$, Anish Kanesa-Thasan ${ }^{1}$ \\ Ann C. McKee ${ }^{1,2,3}$, Richard E. Fine ${ }^{1,2,4}$, John M. Wells ${ }^{1,2}$, Peter J. Morin ${ }^{1,2,4}$ \\ ${ }^{1}$ Geriatric Research Education and Clinical Center, Bedford VA Hospital, Bedford, USA; \\ *Corresponding Author: sullivan95@gmail.com \\ ${ }^{2}$ Department of Neurology, Boston University School of Medicine, Boston, USA \\ ${ }^{3}$ Department of Pathology, Boston University School of Medicine, Boston, USA \\ ${ }^{4}$ Department of Biochemistry, Boston University School of Medicine, Boston, USA
}

Received 29 May 2013; revised 5 July 2013; accepted 18 July 2013

Copyright (C) 2013 Christopher P. Sullivan et al. This is an open access article distributed under the Creative Commons Attribution License, which permits unrestricted use, distribution, and reproduction in any medium, provided the original work is properly cited.

\section{ABSTRACT}

Wnt signaling has been implicated in Alzheimer's disease (AD) pathogenesis, but no studies have described Wnt signaling in aging brain. Phosphorylation of the Wnt co-receptor, lowdensity lipoprotein receptor-related protein 6 (Lrp6), is a sensitive indicator of Wnt ligandreceptor interaction and canonical Wnt signaling. We report that in aged human temporal lobe, the phospho-Lrp6 (pLrp6) epitope localizes to neurons in the entorhinal cortex (EC), the dentate gyrus (DG), and the hippocampal formation, especially in the CA3 field. Activated Lrp6 is detected in neuronal soma and in neuronal processes, particularly in the mossy fiber terminals in the stratum lucidum of CA3. These three regions and their connectivity represent the afferent arm of the major hippocampal circuit. In the DG, cells positive for pLrp6 include Type 1 and Type 2 hippocampal progenitor cells. Overall, these data indicate regional Wnt receptor activation in the human hippocampus that is most prominent in the cells comprising the afferent arm of the major hippocampal circuit that is associated with learning and memory functions. These findings are consistent with data from rodent studies which suggest an important role for Wnts in adult neurogenesis in the human DG. We speculate that Wnt signaling may be an activity-dependent trophic influence in the hippocampus.

Keywords: Wnt; LRP6; Hippocampus; CA3; Neurogenesis; Alzheimer's

\section{INTRODUCTION}

Limited human data suggest that adult neurogenesis in the human hippocampus may differ substantially from rodent models. Doublecortin-expressing cells in human DG diminish in number with advancing age through the tenth decade of life [1] and, unlike rodent models, may be increased in Alzheimer's disease patients compared to age-matched controls [2,3]. One feature of adult neurogenesis that is likely to be preserved across mammalian species is the role of regulatory mechanisms, including Wnt signaling. The neurodevelopment $[4,5]$ and maintenance [6] of the mammalian mesial temporal lobe are dependent on Wnt signaling. Canonical Wnt signaling is thought to be critical for multiple cell fate decisions in both embryonic and adult neurogenesis [7]. These functions of Wnts in adult neurogenesis have been demonstrated using molecular techniques that cannot be applied in human brain.

Recent advances in studies of Wnt signal transduction have identified a unique and sensitive marker of canonical Wnt signaling. Upon Wnt binding to the Wnt receptor complex, the Wnt coreceptor, Lrp6, is phosphorylated at five conserved PPPSPxS motifs on its short intracellular domain (ICD) [8]. The detection of this phosphoepitope 
has been shown to be a highly sensitive indicator of active canonical Wnt signaling that correlates tightly with Super8Topflash (Tcf4) luciferase Wnt-reporter activity in tissue culture models [9].

Here we report that Wnt receptor activation, as indicated by the presence of the pLrp6 epitope, is abundant in cells in the CA3 field, DG and EC of adult human hippocampus. These cells comprise the afferent arm of the major circuit of the hippocampus that is critical for memory and learning [10]. We also find that pLrp6positive cells in the aged human dentate gyrus bear markers of hippocampal progenitor cells and mature neurons. These findings are consistent with rodent data and suggest that Wnt signaling is active in neurons in the adult human hippocampus.

\section{MATERIALS AND METHODS}

\subsection{Subjects and Tissue Preparation}

Human brain tissue used in this study was obtained from the Department of Veteran Affairs VISN1 Neuropathology Center, the Framingham Heart Study (FHS), and the Boston University Alzheimer's Disease Center (ADC). Hippocampal tissue sections from twenty adults over the age of 62 (average age of 81) were evaluated for this study. Brain specimens were fixed in 4\% PLP ( $\mathrm{pH}$ 7.4). After fixation, hippocampus tissue was dehydrated and paraffin-embedded. Postmortem interval (PMI) for brain fixation ranged between 1.5 hours and 24 hours among specimens with an average PMI of 6.8 hours.

\subsection{Western Blot}

HEK 293 cells were grown on 6-well tissue culturetreated dishes (BD Biosciences) and treated with normal or Wnt-conditioned media for various time periods. Cell lysates were collected in RIPA buffer (Pierce) in the presence of phosphatase inhibitors (HALT cocktailPierce). Proteins were separated by polyacrylamide gel electrophoresis and transferred to polyvinylidene fluoride membranes. Membranes were blocked in SuperBlock (Pierce) before incubation with rabbit anti-pLrp6 antibody (Cell Signaling Technologies) overnight. After washing, membranes were treated with HRP-tagged donkey anti-rabbit secondary antibody (Vector Labs) and visualized by chemiluminescence (Pierce).

\subsection{Immunocytochemistry}

HEK 293 cells were grown on chamber slides (BD Biosciences) and treated with normal or Wnt-conditioned media for 12 hours. Cells were washed with PBS and fixed in PBS with 4\% paraformaldehyde for 10 minutes. Cells were permeabilized with PBS containing $0.1 \%$ TX-100 (BioRad) for 3 minutes, and blocked in PBS containing 5\% normal goat serum before incubating with primary antibodies overnight at $4{ }^{\circ} \mathrm{C}$, followed by treatment with fluorescently-labeled secondary antibodies (Invitrogen) as previously described [11].

\subsection{Immunohistochemistry}

$5 \mu \mathrm{m}$ paraffin-embedded formalin-fixed human hippocampal sections were deparaffinized and rehydrated with distilled water. Sections were blocked in SuperBlock reagent (ScyTek) with 5\% serum derived from the species used to raise the secondary antibody. Detection of pLrp6 in tissue sections was carried out using a Ser1490 phosphorylation site-specific primary antibody (Cell Signaling Technologies). For brightfield microscopy, detection of pLrp6-bound primary antibodies was carried out with horse radish peroxidase (HRP)-conjugated secondary antibodies, exposed by enzymatic processing of diaminobenzidine (DAB). Fluorescence confocal microscopy (Leica SP5) was used as previously described [12] to assess colocalization of pLrp6 with primary antibodies bound to NeuN, Sox2, and GFAP (Santa Cruz Biotechnology). Secondary antibodies conjugated with Alexafluor 488, 555 or 633 dyes (Life Technologies) were used to detect bound primary antibodies for fluorescence imaging. To reduce background fluorescence, slides were immersed in lipofuscin-reducing solution [5 $\mathrm{mM} \mathrm{CuSO}_{4}$ in $50 \mathrm{mM}$ ammonium acetate buffer (pH 5)] for 10 minutes before mounting.

\section{RESULTS}

\section{1. pLrp6 Epitope Is Responsive to Wnt3A}

Phosphorylation of Lrp6 occurs in response to the binding of Wnt ligand to the Wnt receptor complex [8]. The specificity of this epitope as a marker of active Wnt signaling in human cells is well established [13] and is demonstrated here by exposing HEK 293 cells to Wnt3A or control conditioned medium. Detection of pLrp6 at various time points by western blot (Figure 1(a)) and immunocytochemistry (Figure 1(b)) was possible only in cells exposed to Wnt3A conditioned medium.

\section{2. pLrp6 in Human Hippocampus}

While anti-pLrp6 antibodies have been used to detect Wnt activity in lysates and cultured cells, the distribution of pLrp6 in human tissues has not been characterized histologically. In the human hippocampus, previous studies have characterized Wnt signaling at the level of Wnt expression and Wnt-dependent gene expression, using in situ hybridization and immunostaining to detect Wnt-dependent transcripts and proteins [1,6]. To detect Wnt signaling in the adult hippocampus at the level of Wnt receptor activation, immunostaining to detect pLrp6 


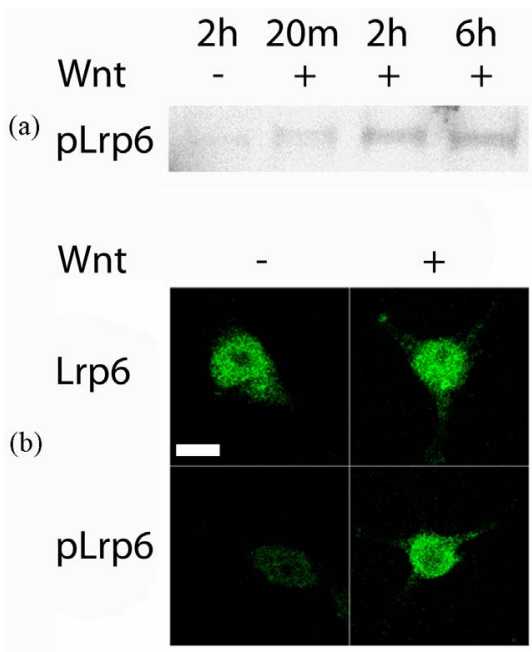

Figure 1. Lrp6 is phosphorylated in response to Wnt treatment. HEK 293 cells were treated with Wnt-conditioned media for the indicated times before analysis by Western blot and ICC. (a) Western blot analysis using anti-pLrp6 antibody was carried out on cell lysates harvested 20 mins, 2 hours and 6 hours after Wnt treatment; (b) Detection of Lrp6 and pLrp6 by immunofluorescence microscopy was carried out on cultured cells treated overnight with Wnt-conditioned media. Scale bar equals $10 \mu \mathrm{m}$.

was carried out. Phosphorylated Lrp6 is detected in cells in the hippocampal formation, DG, and EC (Figure 2). DAB-positive cells (brown) are seen in most cortical regions but are most prevalent in the $\mathrm{EC}$ and in CA3 (bottom panel). In the EC, pLrp6 is seen in large pyramidal neurons and some signal is observed in processes extending from these cells towards the hippocampus. In many cases, a large number of these processes are seen crossing the hippocampal fissure in line with the perforant pathway en route from the EC to the DG and CA3. In some cases, pLrp6 was also seen in neurons in the parasubiculum, and, much less frequently, in the subiculum. Cytoarchitectonic distinction of EC from parasubiculum was difficult in these aged brains, but the location within the parahippocampal gyrus is most consistent with neuronal staining in the EC. DAB detection was diminished in control experiments where the antibody solution was preincubated with an excess of pLrp6 blocking peptide (21st Century Biochemicals) or where a normal rabbit IgG was used in place of anti-pLrp6 (Figure 3).

In the hippocampal formation, many pLrp6-positive neurons were observed throughout the CA fields. The CA3 field; however, contained by far the highest density of DAB-positive pyramidal neurons. As in the EC, many pLrp6-enriched neuronal processes were observed in the (a)

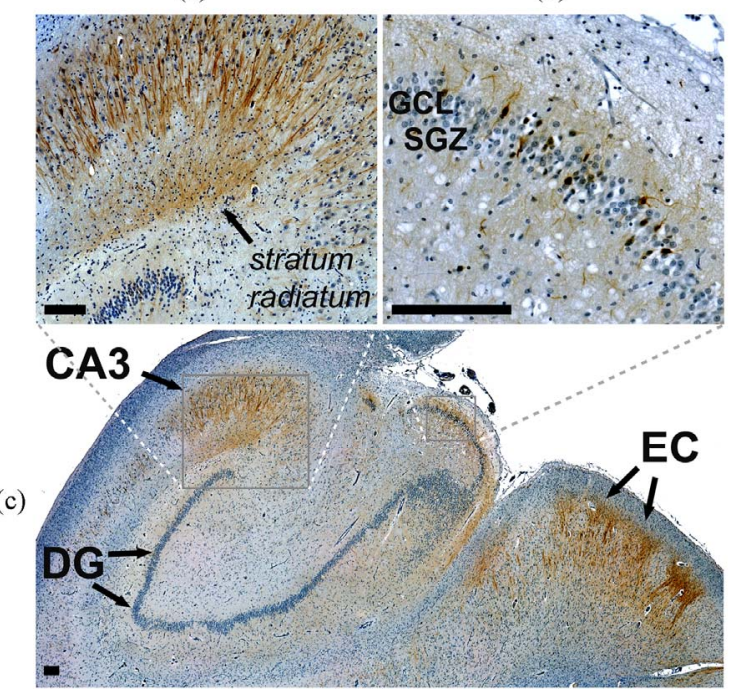

Figure 2. Cells of the hippocampal DG, CA3 and EC harbor pLrp6. Adult human tissue sections were immunostained using anti-pLrp6 antibody and visualized by DAB treatment (brown color). A cross section of the hippocampus is pictured (c) along with enlarged regions highlighting the mossy fibers of CA3 (a) and granule cells of the DG (b). DG-dentate gyrus; ECentorhinal cortex; GCL-granule cell layer; SGZsubgranular zone. Scale bars equal $200 \mu \mathrm{m}$.

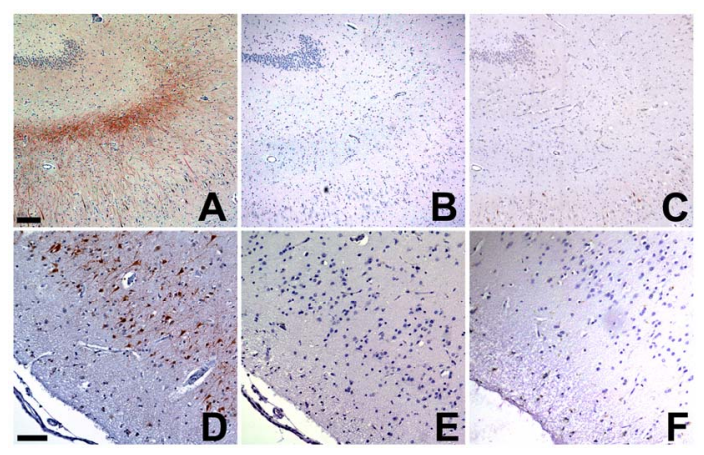

Figure 3. Antigen detection is diminished under control conditions. Adult human tissue sections were immunostained using either ((A) and (D)) anti-pLrp6 antibody, ((B) and (E)) anti-pLrp6 antibody preincubated with a 10-molar excess of a peptide corresponding to the phosphorylated epitope of Lrp6 (21st Century Biochemicals) or ((C) and (F)) with a equal concentration of normal rabbit IgG (Vector Labs) used in place of anti-pLrp6. Bound antibodies were visualized using DAB detection. Scale bar equals 75 $\mu \mathrm{m}$.

CA3 field. In addition to processes in the CA3 field proper, pLrp6 was invariably observed in the stratum radiatum (upper left panel) of CA3, an area were the dendritic fields of these cells receive mossy fiber input from the DG.

In the DG, diffuse pLrp6 signal was consistently ob- 
served in the molecular layer. In the granular cell layer (GCL), pLrp6 was found in a variable and patchy distribution with the highest prevalence found near the tips of the superior and inferior blades. In the most densely stained regions, approximately $10 \%-20 \%$ of the cells stained positively for pLrp6 and exhibited variable morphology. Near the subgranular zone (SGZ), pLrp6-positive cells were small with minimal cytoplasm. Cells deeper in the DG and approaching the molecular layer exhibited the morphology characteristic of mature granule cells, with thick proximal dendritic shafts enriched with pLrp6.

\subsection{Neuronal Identity of pLrp6-Positive Cells}

To determine the identity of the pLrp6-positive cells, the co-localization of pLrp6 with established markers of neurons and neural precursors was evaluated. Adult hippocampal tissue sections were subjected to double fluorescence immunostaining for pLrp6 and the neuronal marker NeuN. All pLrp6-positive cells in the EC and CA3, as well as most of the cells in the DG, had a distinctive neuronal morphology. Fluorescence imaging of DG cells (Figure 4(A)) demonstrated that virtually all of the pLrp6-positive cell bodies co-localized with NeuN (red) and bright pLrp6 signal (green) was observed in both the soma and proximal processes. Additionally, we tested the hypothesis that pLrp6 might be associated with neural precursor cells in the DG, a known neurogenic zone [14]. Colocalization of pLrp6 (green) and Sox2 (red), a transcription factor expressed by hippocampal progenitor cells, was examined by laser scanning confocal microscopy in the DG (Figure 4(B)). While many of the Sox2-positive cells in the DG did not express pLrp6, many of the pLrp6-positive cells also expressed Sox2.

Current evidence suggests that two types of hippocampal progenitor cells exist in the DG, Type 1 and Type 2 [15]. In addition to morphological differences, these cell types can be characterized by whether or not they express glial fibrillary acidic protein (GFAP) and hence become mature astrocytes. While both cell types express Sox 2 , Type 2 progenitors also express GFAP and Type 1 cells do not. To determine whether pLrp6-positive cells could be Type 1 or Type 2, triple immunofluorescent staining was carried out on human hippocampal tissue sections to colocalize pLrp6 with both Sox 2 and GFAP. Lrp6 phosphorylation was observed in both Type 1 (Figure 4(C-bottom)) and Type 2 (Figure 4(C-top)) neural progenitors. Thus, our data are suggestive of Wnt recaptor activation in both Type 1 and Type 2 progenitor cells, a result that is consistent with observations of cell proliferation in human DG [14,15].

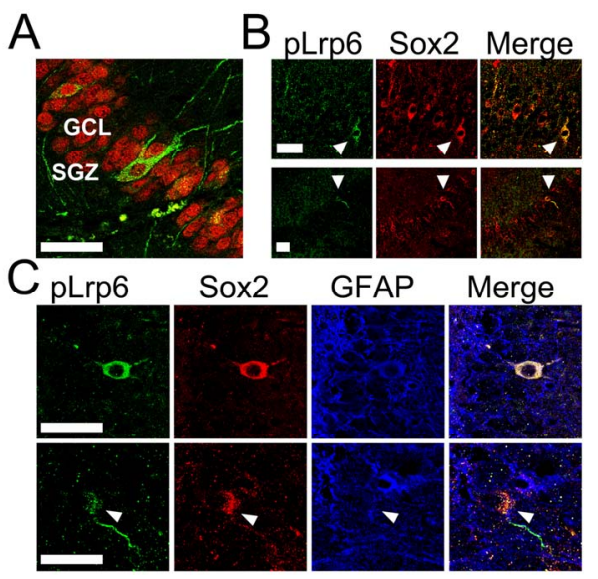

Figure 4. Wnt-activated cells in the human DG colocalize with neuronal and stem cell specific markers. (A) DG cells positive for pLrp6 (green) colocalize with the neuronal marker NeuN (red); (B) A subset of cells expressing Sox2 (red) also contain pLrp6 (green) (areas of colocalization appear in yellow in the merged image); (C-top) A subset of cells expressing pLrp6 (green) and Sox2 (red) also express GFAP (blue) while others (C-bottom) do not. Scale bars equal $50 \mu \mathrm{m}$.

\section{DISCUSSION}

In this study, we demonstrate that pLrp6 is present in post mortem aged human brain and we report three novel findings. These results establish a method for studying Wnt signaling activity in situ, demonstrate regional Wnt signaling within the medial temporal lobe structures, and implicate Wnt signaling in adult neurogenesis in human brain.

Our data suggest that Wnt signaling in the human hippocampus is mostly active in neurons. In the central nervous system (CNS), Wnt signaling has been found in neurons [16], astrocytes in response to brain injury [17], and endothelial cells [18]. Most of the pLrp6-positive cells bodies we identified in EC and CA3 expressed NeuN and most exhibit a distinctly neuronal morphology (Figures 2 and 4). Immunoreactivity was observed in neuronal soma and in straight-shaft processes that conform to established tracts, suggesting a neuronal localization. In the CA3 region, pLrp6 signal may also be associated with synaptic structures. DAB signal in the mossy fibers is somewhat diffuse, and some synaptic bouton-associated immunoreactivity cannot be excluded. Some of the regions of intense cell staining might include endothelial cells, but the morphological features of pLrp6-positive cells are most consistent overall with neuronal cells, and no distinctive features of glial or endothelial cells have been identified.

In the DG, pLrp6-positive cells exhibit a spectrum of 
morphologies. Within the GCL, small cells with limited cytoplasm are often seen near the SGZ, while large cells with a distinctly neuronal morphology (including processes) are seen throughout the DG and often are closer to the molecular layer. Frequently, small pLrp6-positive cells are found in small clusters, suggesting a common lineage. Of the pLrp6-positive cells observed in the DG, many express Sox2, and a subset express both Sox 2 and GFAP. These findings suggest that Wnt receptor activation is present in hippocampal progenitor cells. Current data from rodents support a role for canonical Wnt signaling in multiple stages of neurogenesis, including asymmetric division of radial glial cells and expansion of intermediate precursor cell populations [6]. Our human data are consistent with these findings.

We cannot, however, exclude the possibility that canonical Wnt signaling may be active in these cells due to synaptic activity. Recent studies support a role for activity-dependent Wnt signaling in synaptic remodeling [19], including experiential synaptic remodeling in the dendrites of CA3 neurons [20]. While preliminary experiments did not identify activity-dependent epitopes in these cells (not shown), we are continuing to explore this hypothesis. The presence of the pLrp6 in some mature DG granule cells could also indicate that the activated Wnt receptor is retrogradely transported. Support for such a model stems from reports of trans-synaptic activity of wingless $(\mathrm{Wg})$ at the Drosphila neuromuscular junction where pre-synaptic Wnt ligand secretion is tightly coupled, through a mechanism involving exosome secretion [21], to retrograde transport of post-synaptic $\mathrm{Wg}$ receptor (DFr2) [22]. This explanation is consistent with the appearance of pLrp6 in neuronal processes in the EC, DG, and CA3. Finally, these data do not exclude the possibility of cell-autonomous regulation of receptor activation by a ubiquitous Wnt signal in the extra cellular matrix. Further studies will be necessary to determine the mechanisms and function of Wnt signaling in human neurons.

The regional distribution of pLrp6 coincides with the afferent arm of the major hippocampal circuit that has been implicated in learning and memory [10]. This circuit includes the EC, the DG and the CA3 field of the hippocampal formation. Our data show that mature pLrp6positive neurons are found in each of these regions at a prevalence that exceeds surrounding regions. We also found pLrp6 in neuronal processes, including in the perforant pathway, mossy fibers, and the molecular layer of the DG. These fibers comprise the major tracts linking the EC to the CA3. In contrast, less pLrp6 immunoreactivity is seen in the efferent arc of this circuit: the Schaffer collaterals, CA1, and subiculum. It is important to emphasize that there is some pLrp6 signal in CA1, but much less than in CA3. The CA1 signal may reflect EC neurons projecting directly to CA1. The significance of relatively robust Wnt signaling in hippocampal circuitry that is well known for plasticity is not immediately clear. We are inclined to interpret these findings as supporting a role for Wnt signaling in synaptic remodeling. One caveat relates to studies of "activity-dependent" findings in post mortem tissue. In this setting, activity-dependent signaling could be related to neural activity at or near the time of death and/or be related to unique circuits within the EC-DG-CA3 circuit. Such data may be distorted in human autopsy tissue due to the post mortem interval measured in hours. Further experiments in mouse brain will be necessary to investigate activity-dependent Wnt signaling in the hippocampus. These studies have potential implications for $\mathrm{AD}$ [23] where deranged Wnt signaling in the aged hippocampus could be associated with synaptic injury and spread to interconnected brain regions. The distribution of pLrp6 that we observe corresponds to that of neuronal circuits is known to be injured early in the disease process and that are associated with amnestic symptoms that characterize early age-related memory deficits [24]. Additional studies aimed at evaluating Wnt signaling activity in AD brain will be necessary to establish the significance of these neuropathological findings.

\section{ACKNOWLEDGEMENTS}

This material is based upon work supported by the Department of Veterans Affairs, Veterans Health Administration, Office of Research and Development, Biomedical Laboratory Research and Development.

\section{REFERENCES}

[1] Knoth, R., Singec, I., Ditter, M., Pantazis, G., Capetian, P., Meyer, R.P., Horvat, V., Volk, B. and Kempermann G. (2010) Murine features of neurogenesis in the human hippocampus across the lifespan from 0 to 100 years, PLoS One, 5, Article ID: e8809.

http://dx.doi.org/10.1371/journal.pone.0008809

[2] Boekhoorn, K., Joels, M. and Lucassen, P.J. (2006) Increased proliferation reflects glial and vascular-associated changes, but not neurogenesis in the presenile Alzheimer hippocampus. Neurobiology of Disease, 24, 1-14. http://dx.doi.org/10.1016/j.nbd.2006.04.017

[3] Jin, K., Peel, A.L., Mao, X.O., Xie, L., Cottrell, B.A., Henshall, D.C. and Greenberg, D.A. (2004) Increased hippocampal neurogenesis in Alzheimer's disease. Proceedings of the National Academy of Sciences of the United States of America, 101, 343-347. http://dx.doi.org/10.1073/pnas.2634794100

[4] Lee, S.M., Tole, S., Grove, E. and McMahon, A.P. (2000) A local Wnt-3a signal is required for development of the mammalian hippocampus. Development, 127, 457-467.

[5] Zhou, C.J., Borello, U., Rubenstein, J.L. and Pleasure, S.J. (2006) Neuronal production and precursor proliferation 
defects in the neocortex of mice with loss of function in the canonical Wnt signaling pathway. Neuroscience, 142, 1119-1131. http://dx.doi.org/10.1016/j.neuroscience.2006.07.007

[6] Lie, D.C., Colamarino, S.A., Song, H.J., Desire, L., Mira, H., Consiglio, A., Lein, E.S., Jessberger, S., Lansford, H., Dearie, A.R. and Gage, F.H. (2005) Wntsignalling regulates adult hippocampal neurogenesis. Nature, 437, 13701375. http://dx.doi.org/10.1038/nature04108

[7] Munji, R.N., Choe, Y., Li, G., Siegenthaler, J.A. and Pleasure, S.J. (2011) Wnt signaling regulates neuronal differentiation of cortical intermediate progenitors. The Journal of Neuroscience, 31, 1676-1687.

http://dx.doi.org/10.1523/JNEUROSCI.5404-10.2011

[8] MacDonald, B.T., Yokota, C., Tamai, K., Zeng, X. and He, $X$. (2008) Wnt signal amplification via activity, cooperativity, and regulation of multiple intracellular PPPSP motifs in the Wnt co-receptor LRP6. The Journal of Biological Chemistry, 283, 16115-16123. http://dx.doi.org/10.1074/jbc.M800327200

[9] Piao, S., Lee, S.H., Kim, H., Yum, S., Stamos, J.L., Xu, Y., Lee, S.J., Lee, J., Oh, S., Han, J.K., Park, B.J., Weis, W.I. and Ha, N.C. (2008) Direct inhibition of GSK3beta by the phosphorylated cytoplasmic domain of LRP6 in Wnt/ beta-catenin signaling. PLoS One, 3, Article ID: e4046.

[10] Squire, L.R., Stark, C.E. and Clark, R.E. (2004) The medial temporal lobe. Annual Review of Neuroscience, 27, 279-306. http://dx.doi.org/10.1146/annurev.neuro.27.070203.14413 $\underline{0}$

[11] Sullivan, C.P., Jay, A.G., Stack, E.C., Pakaluk, M., Wadlinger, E., Fine, R.E., Wells, J.M. and Morin, P.J. (2011) Retromer disruption promotes amyloidogenic APP processing. Neurobiology of Disease, 43, 338-345. http://dx.doi.org/10.1016/j.nbd.2011.04.002

[12] Sullivan, C.P., Berg, E.A., Elliott-Bryant, R., Fishman, J.B., McKee, A.C., Morin, P.J., Shia, M.A. and Fine, R.E. (2011) Pyroglutamate-A\&\#946; 3 and 11 colocalize in amyloid plaques in Alzheimer's disease cerebral cortex with pyroglutamate-A\&\#946; 11 forming the central core. Neuroscience Letters, 505, 109-112.

[13] Zeng, X., Tamai, K., Doble, B., Li, S., Huang, H., Habas, R., Okamura, H., Woodgett, J. and He, X. (2005) A dualkinase mechanism for Wnt co-receptor phosphorylation and activation. Nature, 438, 873-877. http://dx.doi.org/10.1038/nature04185

[14] Eriksson, P.S., Perfilieva, E., Bjork-Eriksson, T., Alborn, A.M., Nordborg, C., Peterson, D.A. and Gage, F.H. (1998) Neurogenesis in the adult human hippocampus. Nature
Medicine, 4, 1313-1317. http://dx.doi.org/10.1038/3305

[15] Zhao, C., Deng, W. and Gage, F.H. (2008) Mechanisms and functional implications of adult neurogenesis. Cell, 132, 645-660.

http://dx.doi.org/10.1016/j.cell.2008.01.033

[16] L'Episcopo, F., Serapide, M.F., Tirolo, C., Testa, N., Caniglia, S., Morale, M.C., Pluchino, S. and Marchetti, B. (2011) A Wnt1 regulated Frizzled-1/beta-Catenin signaling pathway as a candidate regulatory circuit controlling mesencephalic dopaminergic neuron-astrocyte crosstalk: Therapeutical relevance for neuron survival and neuroprotection. Molecular Neurodegeneration, 6, 49. http://dx.doi.org/10.1186/1750-1326-6-49

[17] White, B.D., Nathe, R.J., Maris, D.O., Nguyen, N.K., Goodson, J.M., Moon, R.T. and Horner, P.J. (2010) Beta-catenin signaling increases in proliferating NG2+ progenytors and astrocytes during post-traumatic gliogenesis in the adult brain. Stem Cells, 28, 297-307.

[18] Liebner, S. and Plate, K.H. (2010) Differentiation of the brain vasculature: The answer came blowing by the Wnt. Journal of Angiogenesis Research, 2, 1.

[19] Budnik, V. and Salinas, P.C. (2011) Wnt signaling during synaptic development and plasticity. Current Opinion in Neurobiology, 21, 151-159. http://dx.doi.org/10.1016/j.conb.2010.12.002

[20] Farias, G.G., Alfaro, I.E., Cerpa, W., Grabowski, C.P., Godoy, J.A., Bonansco, C. and Inestrosa, N.C. (2009) Wnt-5a/ JNK signaling promotes the clustering of PSD-95 in hippocampal neurons. Journal of Biological Chemistry, 284, 15857-15866. http://dx.doi.org/10.1074/jbc.M808986200

[21] Korkut, C., Ataman, B., Ramachandran, P., Ashley, J., Barria, R., Gherbesi, N. and Budnik, V. (2009), Trans-synaptic transmission of vesicular Wnt signals through Evi/ Wntless. Cell, 139, 393-404. http://dx.doi.org/10.1016/j.cell.2009.07.051

[22] Speese, S.D. and Budnik, V. (2007) Wnts: Up-and-coming at the synapse. Trends in Neurosciences, 30, 268-275. http://dx.doi.org/10.1016/j.tins.2007.04.003

[23] Iqbal, K. and Grundke-Iqbal, I. (2011) Opportunities and challenges in developing Alzheimer disease therapeutics. Acta Neuropathologica, 122, 543-549. http://dx.doi.org/10.1007/s00401-011-0878-Z

[24] Yassa, M.A., Mattfeld, A.T., Stark, S.M. and Stark, C.E. (2011) Age-related memory deficits linked to circuitspecific disruptions in the hippocampus. Proceedings of the National Academy of Sciences of the United States of America, 108, 8873-8878. http://dx.doi.org/10.1073/pnas.1101567108 